\title{
ON ALGEBRAIC GROUPS DEFINED BY NORM FORMS OF SEPARABLE EXTENSIONS
}

\section{TAKASHI ONO}

Let $K$ be any field, and $L$ a separable extension of $K$ of finite degree. $\quad L$ has a structure of vector space over $K$, and we shall denote this space by $V$. The space of endomorphisms of $V$ will be denoted by $(V)$. Let $x$ be any element of $L$, and $N(x)$ the norm of $x$ relative to the extension $L / K . \quad N$ is then a function defined on $V$ with values in $K$. We shall call $N$ the norm form on $V$. The multiplicative groups of non-zero elements of $K$ and $L$ will be denoted by $K^{*}$ and $L^{*}$ respectively. Let $H$ be any subgroup of $K^{*}$. Then the elements $z$ of $L^{*}$ such that $N(z) \in H$ form a subgroup of $L^{*}$, which we shall denote by $G_{H}$. On the other hand the elements $s$ of $\&(V)$ such that $N(s x)=\Lambda(s) N(x)$ with $A(s) \in H$ for all $x \in V$, form obviously a subgroup of $G L(V)$, which we shall denote by $\widetilde{G}_{H} . \quad \widetilde{G}_{H}$ becomes an algebraic group if $H=K^{*}$ or $\{1\}$. In case $H=K^{*}, \widetilde{G}_{H}=\widetilde{G}_{K^{*}}$ will mean the group of linear transformations of $V$ leaving semi-invariant the norm form of $L / K$ and in case $H=\{1\}, \quad \widetilde{G}_{H}=\widetilde{G}_{\{1\}}$ will mean the group of linear transformations of $V$ leaving invariant the norm form of $L / K$.

The object of this paper is to investigate the structure of these groups $\widetilde{G}_{H}$, particularly in the cases $H=K^{*}$ and $H=\{1\}$. Our result in most general form reads in Proposition 2, which is obtained under a sole hypothesis that $K$ contains infinitely many elements. Theorems 1 and 2 correspond respectively to the cases $H=K^{*}$ and $H=\{1\}$. Theorem 2 will show in particular that $G_{\{1\}}$ is the algebraic component of $\widetilde{G}_{\{1\}}$, and if $L / K$ is normal, $\widetilde{G}_{\{1\}}$ may be considered as a semi-direct product ${ }^{1)}$ of $G_{\{1\}}$ and the Galois group of $L / K$. Theorem 3 gives the center of $\widetilde{G}_{I I}$.

The significance of the group $G_{\{1\}}$ as an algebraic group was indicated by Chevalley. ${ }^{2)}$ The groups $G_{\{1\}}$ and $\widetilde{G}_{\{1\}}$ may be regarded as analogues of special

Received August 18, 1956.

1) For definition, see p. 127 , footnote 3 ).

2) Théorie des groupes de Lie: Vol. 2, Hermann, Paris, 1951, p. 170. We shall quote this book as C. II. We shall also quote Vol. 3 (1955) of the series as C. III. 
orthogonal and orthogonal groups respectively. The groups $G_{H}$ and $\widetilde{G}_{H}$ have arithmetic meanings when $K$ is the field of rational numbers, and we have in mind to investigate further arithmetic applications on later occasion.

Now, we denote by $\&$ the group of automorphisms of $L$ leaving invariant each element of $K$. For simplicity we shall call $\$$ the automorphism group of $L / K$. Obviously $\&$ is a subgroup of $G L(V)$. Each element $z \in L$ defines an endomorphism $\mu(z)$ of $V$ by

$$
\mu(z)(x)=z x, \quad x \in V .
$$

The mapping $\mu$ is clearly an isomorphism of $V$ into $\&(V)$, and we have $\mu\left(L^{*}\right)=\mu(V) \frown G L(V)$. It follows at once that $\mu\left(G_{H}\right) \subset \widetilde{G}_{H}$ and $\mathbb{S} \subset \widetilde{G}_{\{1\}}$. We shall set $G=G_{\{1\}}$ and $\widetilde{G}=\widetilde{G}_{\{1\}}$.

Proposition 1. For any $H \subset K^{*}$, we have $\mathbb{G}_{\frown} \cap \mu\left(G_{H}\right)=\{\varepsilon\}$ where $\varepsilon$ is the identity endomorphism in (5) $V)$.

Proof. Take an element $\mu(z) \in \mathbb{B} \cap \mu\left(G_{H}\right)$. Then, it follows that $1=\mu(z)(1)=z$ and $\mu(z)=\varepsilon$.

Proposition 2. Assume that $K$ is an infinite field. Then, for any $H \subset K^{*}$, we have $\widetilde{G}_{H}=\mu\left(G_{H}\right) \mathbb{S}$.

Proof. Let $N$ be a Galois extension of $K$ containing $L$. We denote by $\mathfrak{S}$ and $\mathscr{R}$ the Galois groups of $N / K$ and $N / L$ respectively. Let $\sigma(\omega), \omega \in N, \sigma \in \mathscr{H}$ be a normal base of $N / K$. By some representatives $\tau_{i}, 1 \leqq i \leqq n$, of right cosets of $\mathscr{S}$ modulo $\mathfrak{R}$, we put $\eta_{i}=\sum_{\sigma \in \mathscr{R}} \sigma \tau_{i}(\omega), 1 \leqq i \leqq n$, where we set $\tau_{1}=1$, the identity in $\mathfrak{S}$. It follows at once that $\eta_{i}$ form a base of $L / K$. Let $V^{N}$ be the scalar extension of $V$ with respect to $N$. We define elements $\lambda_{j}, 1 \leqq j \leqq n$, in the dual space $\left(V^{N}\right)^{*}$ by putting $\lambda_{j}\left(\eta_{i}\right)=\tau_{j}\left(\eta_{i}\right), 1 \leqq i, j \leqq n$. Since $\operatorname{det}\left(\tau_{j}\left(\eta_{i}\right)\right) \neq 0, \lambda_{j}, 1 \leqq j \leqq n$, form a base of $\left(V^{N}\right)^{*}$. For $x=\sum_{i} x_{i} \eta_{i} \in V$, we have $N(x)=\prod_{j}\left(\sum_{i} x_{i} \tau_{j}\left(\eta_{i}\right)\right)=\prod_{j} \lambda_{j}(x)$. We set $(\eta(s) \lambda)(x)=\lambda(s x)$ for $s \in \&\left(V^{N}\right)$, $\lambda \in\left(V^{N}\right)^{*}, x \in V^{N}$. Then clearly we have $\eta(s) \lambda \in\left(V^{N}\right)^{*}$ and we get $\eta(s) \lambda_{j}$ $=\sum_{k} a_{k j} \lambda_{k}$ with $a_{k j} \in N$. Now let $s$ be any element of $\widetilde{G}_{H}$. Then, we have $\prod_{j}\left(\sum_{k} a_{k j} \lambda_{k}\right)(x)=\Lambda(s) \prod_{j} \lambda_{j}(x)$ for all $x \in V$. As $K$ contains infinitely many elements, this implies that $\prod_{j}\left(\sum_{k} a_{k j} \lambda_{k}\right)=\Lambda(s) \prod_{j} \lambda_{j}$ in the symmetric algebra on $V^{N}$. Thus, by a well known theorem on the decomposition of polynomials, there exists an integer $k(j)$ for each $j$ such that $k(j) \neq k\left(j^{\prime}\right)$ if $j \neq j^{\prime}$, and 
$a_{k j} \neq 0$ if and only if $k=k(j)$; Therefore we have $\eta(s) \lambda_{j}=a_{j} \lambda_{k(j)}, a_{j} \in N^{*}$. In particular for $j=1$, we get $s\left(\eta_{i}\right)=\lambda_{1}\left(s \eta_{i}\right)=\left(\eta(s) \lambda_{1}\right)\left(\eta_{i}\right)=a_{1} \tau_{k(1)}\left(\eta_{i}\right), 1 \leqq i \leqq n$. Since we have $\sum_{i} \tau_{k(1)}\left(\gamma_{i}\right)=\sum_{\sigma \in \mathfrak{S}} \omega^{\sigma} \in K^{*}$ and $s\left(\eta_{i}\right) \in L$, this implies that $a_{1} \in L$ and we see that $\tau_{k(1)} \in \mathbb{S}$. As we have $N(s x)=N\left(a_{1} \tau_{k(1)}(x)\right)=N\left(a_{1}\right) N(x)$, it follows that $N\left(a_{1}\right)=\Lambda(s) \in H$. Thus we have $s=\mu\left(a_{1}\right) \tau_{k(1)} \in \mu\left(G_{H}\right) \&$. q.e.d.

As an immediate cosequence of the two propositions, we get the following

Corollary. If $K$ contains infinitely many elements, $\widetilde{G}_{H}$ is a semi-direct product of $\mu\left(G_{H}\right)$ and $\left(\mathbb{S}^{3)}\right.$.

Suppose now $K$ is infinite. We shall restrict our attention to the case where $H$ is algebraic, i.e. $H=K^{*}$ or $H=\{1\}$. The mapping $\mu$, which is a linear isomorphism of $V$ onto $\mu(V)$, gives also a homeomorphism of $V$ onto $\mu(V)$ in the sense of Zariski-topology, and every closed set in $\mu(V)$ is also closed in $\&(V)$ since $\mu(V)$, being a linear subspace of $\&(V)$, is closed in $E(V)$. Also each irreducible set of $V$ is mapped on an irreducible set of $\mu(V)$ and vice versa, and every irreducible set in $\mu(V)$ is irreducible in $\&(V){ }^{4)}$ Since $\mu\left(L^{*}\right)=\mu(V) \frown G L(V), \mu\left(L^{*}\right)$ is an algebraic group on $V$ and is irreducible as an open subset in $\mu(V)$. By Proposition 2, the group $\widetilde{G}_{K^{*}}$ has $\mu\left(G_{K^{*}}\right)=\mu\left(L^{*}\right)$ as a subgroup of a finite index. Thus we get by the above corollary the following

THEOREM 1. Let $K$ be an infinite field and $L / K$ a separable extension of finite degree. Then, the group $\widetilde{G}_{K}^{*}$ of all linear transformations of $L$ over $K$ which leave semi-invariant the norm form of $L / K$ is algebraic on the vector space $L$ over $K$ and $\mu\left(L^{*}\right)$ is the algebraic component of $\widetilde{G}_{K^{*}}, \mu$ being defined by (1). Furthermore $\widetilde{G}_{K^{*}}$ is the semi-direct product of $\mu\left(L^{*}\right)$ and $\$ \$ S$, where $\$ \$ S$ is the automorphism group of $L / K$.

Next, we shall consider the group $\widetilde{G}$, i.e. the group of all linear transformations of $V$ leaving invariant the norm form of $L / K$. Of course $\widetilde{G}$ is an algebraic group on $V . \quad G$ being closed in $V, \mu(G)$ is also algebraic. We define a raitonal representation $\tilde{N}$ of $\mu\left(L^{*}\right)$ by $\tilde{N}(\mu(x))=N(x), x \in L^{*}$. Let $H$ be the smallest algebraic group containing $\tilde{N}\left(\mu\left(L^{*}\right)\right)$. Then, $H$ is irreducible

3) We say that a group $G$ is a semi-direct product of a normal subgroup $N$ and a subgroup $H$ if we have $G=N \cdot H$ and $N \cap H=\{e\}, e$ being the identity in $G$. We see that $\mu\left(G_{H}\right)$ is normal in $\widetilde{G}_{H}$ by the relation $\sigma \mu(z) \sigma^{-1}=\mu(\sigma(z)), z \in L, \sigma \in \mathbb{\$}$,

4) Cf. C. III. Chap. VI $\S 1$, 
and $H=\{1\}$ or $H=K^{*}$. But as $K$ is infinite, $\widetilde{N}\left(\mu\left(L^{*}\right)\right) \varsubsetneqq\{1\}$ and we have $H=K^{*}$. Since $\mu(G)$ is the kernel of the representation $\tilde{N}$, it follows that $\operatorname{dim}_{K} \mu(G) \leqq n-1$, where $n=[L: K]^{5)}$ On the other hand, we shall define a homomorphism $\rho$ of $L^{*}$ into itself by $\rho(x)=x^{-n} N(x), x \in L^{*}$. Obviously we have $\rho\left(L^{*}\right) \subset G$ and $\rho$ induces a rational representation $\widetilde{\rho}$ of $\mu\left(L^{*}\right)$ in $\mu(G)$ by $\widetilde{\rho}(\mu(x))=\mu(\rho(x)), x \in L^{*}$. We denote by $H$ the smallest algebraic group containing $\widetilde{\rho}\left(\mu\left(L^{*}\right)\right)$. If we take an algebraically closed field $M$ containing $K$, then we have $\left.H^{M}=(\widetilde{\rho})^{M}\left(\mu\left(L^{*}\right)^{M}\right)\right)^{6)} \quad$ We denote by $\mu^{M}$ the unique extension of $\mu$ to $V^{M}=L^{M}$. Let $\left(L^{M}\right)^{*}$ be the group of all invertible elements of $L^{M}$ which is considered as an algebra over $M$. It follows that $\mu^{M}\left(\left(L^{M}\right)^{*}\right)=\mu^{M}\left(L^{M}\right) \frown G L\left(V^{M}\right)$ $=\overline{\mu(L)} \frown G L\left(V^{M}\right)=\overline{\mu\left(L^{*}\right)} \frown G L\left(V^{M}\right)=\mu\left(L^{*}\right)^{M}$, where $\overline{\mu(L)}$ and $\overline{\mu\left(L^{*}\right)}$ mean the closures of $\mu(L)$ and $\mu\left(L^{*}\right)$ in $V^{M}$ respectively. Let $\rho^{M}$ be the unique extension of $\rho$ to $\left(L^{M}\right)^{*}$. It follows that $\operatorname{dim}_{K} H=\operatorname{dim}_{M} H^{M}=\operatorname{dim}_{M}(\widetilde{\rho})^{M}\left(\mu\left(L^{*}\right)^{M}\right)=$ $\left.\operatorname{dim}_{M} \mu^{M}\left(\rho^{M}\left(L^{M}\right)^{*}\right)\right)=\operatorname{dim}_{M} \rho^{M}\left(\left(L^{M}\right)^{*}\right)$. Since $L / K$ is separable and $M$ is algebraically closed, we have $V^{M}=L^{M}=M e_{1}+\ldots+M e_{n}$ with pimitive idempotents $e_{i}, 1 \leqq i \leqq n$. Let $x=\sum_{i} x_{i} e_{i}$ be in the kernel of the homomorphism $\rho^{M}$. From the relation $\left.N^{M}(x)=x^{n},{ }^{7}\right)$ it follows that $\left(x_{1} \cdots x_{n}\right) 1=\left(x_{1} \cdots x_{n}\right)\left(e_{1}+\cdots\right.$ $\left.+e_{n}\right)=x_{1}^{n} e_{1}+\ldots+x_{n}^{n} e_{n}$ and that $x_{1}^{n}=\ldots=x_{n}^{n}$. Therefore the kernel of $\rho^{M}$ is of 1 -dimension over $M$, as it has $M^{*}$ as a subgroup of finite index, and so the kernel of $(\widetilde{\rho})^{M}$ is also of 1-dimension over $M$. $\quad M$ being algebraically closed, it follows that $\operatorname{dim}_{K} H=\operatorname{dim}_{M}(\tilde{\rho})^{M l}\left(\mu\left(L^{*}\right)^{M}\right)=n-1{ }^{8)} \quad$ Since $H$ is contained in $\mu(G)$, we get at once $\operatorname{dim}_{K} \mu(G) \geqslant n-1$. Hence, we have $\operatorname{dim}_{K} \mu(G)$ $=n-1$. Now, let $\mu\left(G_{1}\right)$ be the algebraic component of $\mu(G)$ and let $G=G_{1}+$ $\ldots+G_{r}$ be the decomposition of $G$ into the cosets modulo $G_{1}$. Thus each $G_{i}$ is irreducible and $\operatorname{dim}_{K} G_{i}=n-1$. Let $\mathfrak{\$}_{i}, 1 \leqq i \leqq r$, be prime ideals of the polynomial ring $K\left[X_{1}, \ldots, X_{n}\right]$ associated to $G_{i}$ respectively. As is well known each $\mathfrak{P}_{i}$ is principal: $\mathfrak{P}_{i}=\left(P_{i}(X)\right), X=\left(X_{1}, \ldots, X_{n}\right)$. Obviously the ideal $\mathfrak{l}=\mathfrak{P}_{1} \frown \ldots \frown \mathfrak{P}_{r}=\mathfrak{P}_{1} \cdots \mathfrak{P}_{r}$ is associated to $G$. On the other band, every element in $G$ satisfies the equation $F(X)=\prod_{\jmath}\left(\sum_{i} X_{i} \tau_{j}\left(\eta_{i}\right)\right)-1=0$, where

5) C. II. Chap. II. $\$ 6$. Prop. 8. If the characteristic of $K$ is zero, we get $\operatorname{dim}_{K} \mu(G)=$ $n-1$ by C. II. Chap. II. $\$ 14$. Théorème 12 .

6) C. II. Chap. II. §5. Prop. 4, §7. Prop. 2. Cor. 1.

7) $N^{M}$ means the extension of $N$ to $V^{M}$. It is also the norm of the algebra $L^{M}$ over $M$ with respect to the regular representation.

8) C. II. Chap. II. \$6. Prop. 8. Cor. 
$\tau_{j}, \gamma_{i}$ have the same meaning as in Proposition 2. Since $F(X)+1$ splits into the product of different $n$ linear factors in the algebraic closure of $K, F(X)$ is an irreducible polynomial. Since $F(X) \in \mathfrak{A}$, we have $r=1$ and it follows that $\mathfrak{U}=\mathfrak{P}_{1}=(F(X))$ is the associated ideal to $G$. Thus $G$, or $/ \ell(G)$, is irreducible and we get the following

THEOREM 2. Let $K$ be an infinite field, and $L / K$ a separable extension of finite degree $n$. Then, the group $\widetilde{G}$ of all linear transformations of $L$ over $K$ which leave invariant the norm form of $L / K$ is an algebraic group of dimension $n-1$ and $\mu(G)$ is the algebraic component of $\widetilde{G}, \mu$ being defined by (1). Furthermore $\widetilde{G}$ is the semi-direct product of $\mu(G)$ and $\mathbb{S}$, where $\mathbb{B}$ is the automorphism group of $L / K$.

Lastly, we shall determine the center of the $\widetilde{G}_{H}$ defined over an arbitrary field $K$.

Proposition 3. Let $K$ be an arbitrary field and $L / K$ a separable extension of degree $n$. Then, there exists a base $\omega_{i}, 1 \leqq i \leqslant n$ of $L / K$ with $N\left(\omega_{i}\right)=1$.

Proof. Suppose first that $K$ is infinite. Let $L(G)$ be the linear closure of $G$ in $V$. Clearly we have $\operatorname{dim}_{K} L(G) \geq \operatorname{dim}_{K} G=n-1$. (Theorem 2). Since G is irreducible and closed and is not a linear space, $L(G)$ must be the whole space $V .^{9)} \quad$ Next, suppose that $K$ is a finite field with $q$ elements. Thus, the number of elements in $\mathrm{G}$ is $=\left(q^{n}-1\right) /(q-1)$. Let $r$ be the dimension of $L(\mathrm{G})$. Then, we have $\left(q^{n}-1\right) /(q-1) \leqq q^{r}$. From this, it follows that $q^{r}(q-1)=q^{r+1}-q$ ə $q^{n}-1>q^{n}-q$ and $r+1>n$, namely $r=n$. Therefore we have again $L(\mathrm{G})=V$. This proves our proposition.

TheOREM 3. Let $K$ be an arbitrary field and $L / K$ a separable extension of degree $n$. Then the center of $\widetilde{G}_{I I}$ is the image of the group $W_{H}=\left\{a ; a \in \mathrm{G}_{I I}\right.$, $\sigma(a)=a, \sigma \in \mathbb{B}\}$ by the isomorphism $\mu$ defined by (1).

Proof. Let $\zeta$ be any èlement of the center of $\widetilde{G}_{H}$. Let $\omega_{i}$ be a base of $L / K$ with $N\left(\omega_{i}\right)=1,1 \leqq i \leqq n$ (Proposition 3 ). As we have $G \subset \mathrm{G}_{H}, 5$ must commute with $\mu\left(\omega_{i}\right)$ and it must commute with all $\mu(z), z \in L$. Thus it follows that $(\zeta \mu(z))(1)=\zeta(z)=\mu(z) \zeta(1)=z \zeta(1)$. Hence, it follows that $\zeta(z)$ $=\alpha z$ and $\alpha=\zeta(1) \in L^{*}$. On the other hand, $\zeta$ must commute with each $\sigma \in \mathbb{B}$,

9) C.. III. Chap. VI. \$1 Prop. 14, 
Thus, we have $\zeta \sigma(1)=\alpha=\sigma \zeta(1)=\sigma(\alpha)$. Since $\zeta \in \widetilde{G}_{H}$, we get $N(\alpha) \in H$. Conversely, it is easy to see that any $\mu(a)$ with $a \in W_{H}$ is in the center of $\widetilde{G}_{H}$ either by Proposition 2 or by the fact that every $a \in W_{H}$ is an element in $K$ if $K$ is finite.

Corollary. Under the same assumption as in Theorem 3, suppose that $L / K$ is a Galois extension. Then the center of $\widetilde{G}_{H}$ is the image of $W_{H}=$ $\left\{a, a \in K^{*}, a^{n} \in H\right\}$.

Remark 1. We can define the norm form for any algebraic extension $L / K$ of finite degree by means of the regular representation. E.g. if $\mathrm{L} / K$ is a purely inseparable extension of degree $p^{f}$, where $p$ is the characteristic of $K$, we have $N(x)=x^{p^{f}}, x \in L$ and we see at once that $\mu(\mathrm{G})=\widetilde{G}=\{\varepsilon\}$. Thus, we have a simple example showing that the dimension of the kernel of a rational representation $\rho$ of an algebraic group $G$ is strictly smaller than the difference of the dimension of $G$ and that of $\rho(G){ }^{10}{ }^{10}$

Remark 2. The conclusion of Proposition 2 does not hold in general if $K$ is a finite field. E.g. let $K=G F(2),[L: K]=3$. Since $K^{*}$ is of order $1, \widetilde{G}_{H}=$ $\widetilde{G}=G L(V)$. Thus, the order of $\widetilde{G}$ is $=\left(2^{3}-1\right)\left(2^{3}-2\right)\left(2^{3}-2^{2}\right)=168{ }^{11)}$ On the other hand, $\mu\left(K^{*}\right)=\mu(G)$ is of order $2^{3}-1=7$. By Proposition 1 , the order of $\mu\left(L^{*}\right) \& S=3.7=21<168$. The center of $\widetilde{G}$ is of order 1 (Theorem 3 , Corollary). Furthermore this $\widetilde{G}$ is simple as is well known. ${ }^{11}$ Thus, it would be of some interest to study the structure of the finite group $\widetilde{G}$ for these cases.

\section{Mathematical Institute}

Nagoya University

10) C.f. C. II. Chap. II. §6. p. 119.

11) C..f. Dickșon, Linear Groups, pp. 77-83, 